\title{
Label-free plasmonic biosensor for rapid, quantitative, and highly sensitive COVID-19 serology: implementation and clinical validation
}

Olalla Calvo-Lozano ${ }^{1}$, Miquel Sierra ${ }^{1}$, Maria Soler ${ }^{1 *}$, M.-Carmen Estévez ${ }^{1 *}$, Luis ChiscanoCamón $^{2,3}$, Adolfo Ruiz-Sanmartin ${ }^{2,3}$, Juan Carlos Ruiz-Rodriguez ${ }^{2,3}$, Ricard Ferrer ${ }^{2,3}$, Juan José González-López ${ }^{4,5,6}$ Juliana Esperalba $^{4,5}$, Candela Fernández-Naval ${ }^{4,5}$, Leticia Bueno ${ }^{7,8,9}$, Ruben López-Aladid $^{7,8,9}$, Antoni Torres ${ }^{7,8}$, Laia Fernández-Barat ${ }^{7,8,9}$, Sarah Attoumani ${ }^{10}$, Rémi Charrel $^{10}$, Bruno Coutard ${ }^{10}$, and Laura M. Lechuga ${ }^{1}$

1. Nanobiosensors and Bioanalytical Applications Group (NanoB2A), Catalan Institute of Nanoscience and Nanotechnology (ICN2), CSIC, CIBER-BBN and BIST, Campus UAB, 08193 Bellaterra, Barcelona, Spain

2. Intensive Care Department, Vall d'Hebron Hospital Universitari, Vall d'Hebron Barcelona Hospital Campus, Barcelona, Passeig Vall d'Hebron 119-129, 08035, Barcelona, Spain

3 Shock, Organ Dysfunction and Resuscitation Research Group, Vall d'Hebron Research Institute (VHIR), Vall d'Hebron Hospital Universitari, Vall d'Hebron Barcelona Hospital Campus, Passeig Vall d'Hebron 119-129, 08035, Barcelona, Spain

4. Clinical Microbiology Department, Vall d'Hebron Hospital Universitari, Vall d'Hebron Barcelona Hospital Campus, Passeig Vall d'Hebron 119-129,08035, Barcelona, Spain

5. Vall d'Hebron Institut de Recerca (VHIR), Vall d'Hebron Barcelona Hospital Campus, Passeig Vall d'Hebron 119-129, 08035 Barcelona, Spain

6. Department of Genetics and Microbiology, Universitat Autònoma de Barcelona, Plaça Cívica, 08193 Bellaterra, Barcelona, Spain

7. Cellex Laboratory, CiberRes (Centro de Investigación Biomédica en Red de Enfermedades Respiratorias, 06/06/0028), Institut d'Investigacions Biomèdiques August Pi i Sunyer (IDIBAPS), Carrer de Roselló 149, 08036 Barcelona, Spain

8. School of Medicine, University of Barcelona, Carrer de Casanova, 143, 08036 Barcelona, Spain

9. Department of Pneumology, Thorax Institute, Hospital Clinic of Barcelona, Carrer de Villarroel, 170, 08036 Barcelona, Spain

10. Unité des Virus Émergents (UVE: Aix-Marseille Univ-IRD 190-Inserm 1207), Marseille, France

*Corresponding authors: $\quad$ M.-Carmen Estevez: mcarmen.estevez@icn2.cat Maria Soler: maria.soler@icn2.cat 


\begin{abstract}
Serological tests are essential for the control and management of COVID-19 pandemic, not only for current and historical diagnostics but especially for surveillance, epidemiological, and acquired immunity studies. Clinical COVID-19 serology is routinely performed by enzymatic or chemiluminescence immunoassays (i.e., ELISA or CLIA), which provide good sensitivities at the expense of relatively long turnaround times and specialized laboratory settings. Rapid serological tests, based on lateral flow assays, have also been developed and widely commercialized, but they suffer from limited reliability due to relatively low sensitivity and specificity. We have developed and validated a direct serological biosensor assay employing proprietary technology based on Surface Plasmon Resonance (SPR). The biosensor offers a rapid -less than 15 min- identification and quantification of SARS-CoV-2 antibodies directly in clinical samples, without the need of any signal amplification. The portable plasmonic biosensor device employs a custom-designed multi-antigen sensor biochip, combining the two main viral antigens (RBD peptide and N protein), for simultaneous detection of human antibodies targeting both antigens. The SPR serology assay reaches detection limits in the low $\mathrm{ng} \mathrm{mL}^{-1}$ range employing polyclonal antibodies as standard, which are well below the commonly detected antibody levels in COVID-19 patients. The assay has also been implemented employing the first WHO approved anti-SARS-CoV-2 immunoglobulin standard. We have carried out a clinical validation with COVID-19 positive and negative samples $(n=120)$ that demonstrates the excellent diagnostic sensitivity (99\%) and specificity (100\%). This positions our biosensor device as an accurate, robust, and easy-to-use diagnostics tool for rapid and reliable COVID-19 serology to be employed both at laboratory and decentralized settings for the management of COVID-19 patients and for the evaluation of immunological status during vaccination, treatment or in front of emerging variants.
\end{abstract}

Keywords Surface Plasmon Resonance Biosensor, serological test, clinical diagnosis, SARSCoV-2, nucleocapsid protein, receptor-binding domain, antibody detection, COVID-19 


\section{Introduction}

It has been over a year since the World Health Organization (WHO) declared COVID-19 (Coronavirus Disease 2019) as a pandemic. The outbreak of this infectious disease, likely originated in the Hubei region (China) in December 2019 and caused by the SARS-CoV-2 virus (Severe Acute Respiratory Syndrome Coronavirus 2), has rapidly spread worldwide, and it is generating unprecedented and devastating consequences at health, social, and economic levels. To date, COVID-19 has affected more than 140 million people, with more than 3 million deaths. ${ }^{1}$ The emergence of an unknown virus with a lack of population's immunity and accurate diagnostic methods, together with the disease peculiarities (i.e., varied symptomatology, or asymptomatology in a significant percentage of the infected people, long incubation times, high transmission rate, etc.), have undoubtedly contributed to ease its unnoticeable spread, and hinder a fast and early detection of many cases. ${ }^{2-4}$

Current standard diagnosis for the detection of an active infection relies on the detection of the SARS-CoV-2 viral genetic material from respiratory samples, mainly by RT-PCR (Reverse Transcription Polymerase Chain Reaction), ${ }^{5,6}$ which provides excellent levels of sensitivity and specificity, but requiring centralized and specialized laboratories, and between 3 to 48 hours to deliver results. To overcome its limitations related to long turnaround times, rapid antigen tests have already been developed and are being employed in many countries as point-of-care test, although their sensitivity and reliability do not reach yet those achieved with genomic molecular assays. ${ }^{78}$ Complementary to the detection of the active infection, serological tests, which detect the presence in blood of immunoglobulins (Ig) generated by the infected host, play an important role in infectious disease surveillance and pandemic management, providing relevant information to estimate the prevalence of the virus and to better understand the dynamics of acquired immunity. In the case of SARS-CoV-2, the immune response is soon triggered, and antibodies are detectable after few days post-infection. First, IgMs appear during the acute infection phase, which decline with time after few days or even months. Then, long-lasting IgGs are generated, as well as IgA antibodies. ${ }^{9}{ }^{10}$ IgGs are expected to remain in the blood stream at significant concentrations for at least months after infection, conferring immunity to the virus. ${ }^{11,12}$ Although serology assays are not suited for systematic detection of the virus, they are very helpful for the diagnosis of past infections (indirect testing), of suspected patients with negative PCR results, in the identification of asymptomatic patients, and also during the development of new vaccines or treatments. ${ }^{11,13,14}$ In addition, serological tests are extremely useful in hospitals for the ICU bed management and the deisolation of post COVID-19 patients (i.e. PCR-positive patients with a positive serological test). Finally, the emergence of SARS$\mathrm{CoV}-2$ variants with increased resistance to sero-neutralization by antibodies induced after 
vaccination or primary infection, makes serological tests a key component for the response to these variants.

The serological assays developed for COVID-19 are based on the identification of IgMs and IgGs (and to a less extent, IgAs), which are specific for most abundant viral antigens including the Spike protein (S1 and S2 subunits, and the receptor-binding domain (RBD)) and the nucleocapsid N protein. Traditional microplate-format immunoassays, such as ELISA (enzymelinked immunosorbent assay) and CLIA (chemiluminescence immunoassay), are widely used in clinics, as they provide high sensitivities, can be automated, and offer multiplexed capabilities, but they require specific equipment and trained personnel in dedicated laboratories and can be time consuming due to sample manipulation and/or long incubation times. ${ }^{15}$ For massive screening, immunochromatographic lateral flow assays (LFA) have been widely spread due to its facile handling and rapid time-to-result response, becoming the most commercialized assays to perform SARS-CoV-2 serology tests. Some of them can differentiate the type of antibody (IgG and/or IgM), and thus provide information regarding the stage of the infection (e.g., acute phase or past infection), but only in a qualitative manner. Although they provide fast results (15min assay) at the point-of-care (POC), few recent studies show that they are not reliable and accurate enough due to their moderate sensitivity (55-90\%). ${ }^{16-18}$ The development of serological assays capable of performing quantitative analysis is critical for some potentially useful scenarios. ${ }^{19}$ These scenarios include monitoring acquired immunity over time, to be able to predict the duration of acquired immunity, evaluate seroconverted patients' plasma for potential reinfusion in other patients, manage the hospital beds and COVID-19 patients isolation, understanding the relationship between antibody levels and severity of the symptoms, to carry out large-scale epidemiology studies for COVID-19 incidence determination, or helping in vaccine development. ${ }^{13,20,21}$

The ongoing pandemic situation, thus, demands advanced analytical tools that overcome aforementioned sensitivity limitations in serology testing, while still facilitating fast, quantitative and reliable detection at the point-of-care. POC optical biosensors are extremely well positioned to fulfil these needs, as they are sensitive techniques capable of performing label-free, direct and quantitative analysis. Plasmon-based technologies, as the Surface Plasmon Resonance (SPR) biosensor, offer remarkable performance and versatility, and have become one of the most consolidated biosensor technologies for biomolecular interactions and clinical diagnostics, ${ }^{22,23}$ with potential for compactness and miniaturization. Moreover, SPR biosensing have been demonstrated for multiple clinical applications in virology, including also serological assays related to dengue virus, ${ }^{24,25}$ Salmonella, ${ }^{26}$ Epstein-Barr virus ${ }^{27}$, and also for the first SARS-CoV ${ }^{28}$. A few preliminary works and perspectives have been recently reported as well for SARS-CoV-2 $2^{29-31}$, advocating for the potential of this technology as POC diagnostic devices. 
We have fully implemented an SPR-based serological test combining RBD and $\mathrm{N}$ viral antigens for the detection of SARS-CoV-2 specific antibodies from human sera (Figure 1). Our SPR biosensor is small and easy to use, offering label-free and real-time monitoring of biomolecular interactions, therefore enabling a one-step quantitative serological assay performed in less than 15 minutes. After an in-depth optimization of the biorecognition interface and bioassay conditions, we have achieved outstanding analytical sensitivity levels in the range of $\mathrm{ng} \mathrm{mL}^{-1}$, well below the common antibody levels in patients, commonly in the $\mu \mathrm{g} \mathrm{mL}^{-1}$ range. ${ }^{32}$ In order to validate our technology, we performed a comprehensive clinical validation with COVID-19 positive and negative samples collected from patients attended in different hospitals, comparing our results to standard and regulated techniques (i.e., ELISA and CLIA) as well as commercial rapid tests based on LFA.

\section{A SARS-CoV-2 structure}

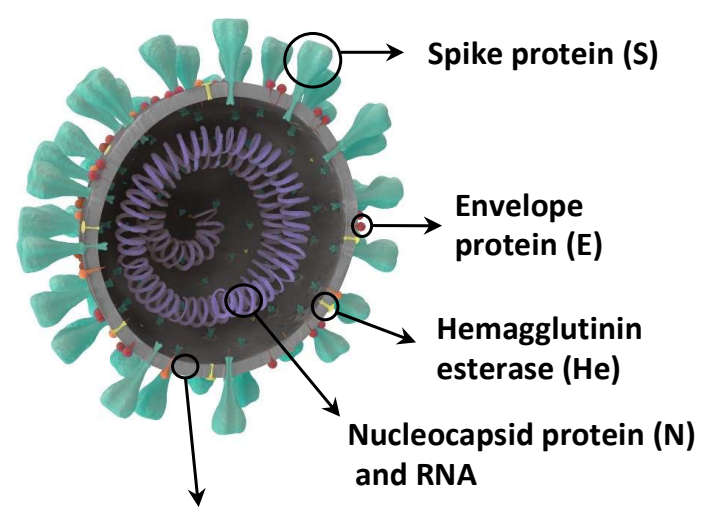

Lipid bilayer membrane

\section{B SPR-based serological test}

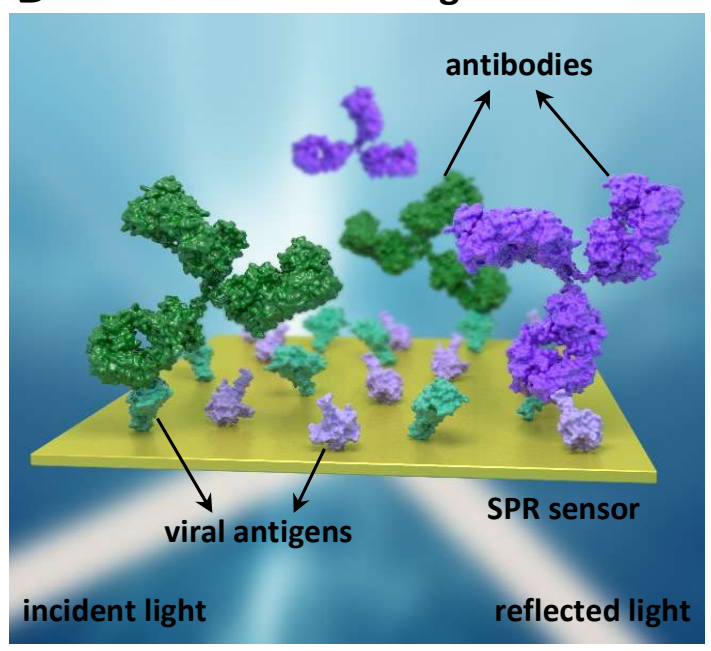

Figure 1. (A) SARS-CoV-2 virus structure; (B) Scheme of the two-antigen co-immobilized sensor biochips employed in the SPR biosensor for COVID-19 serology.

\section{Materials and methods}

\subsection{Chemical and biological reagents}

All the buffer compounds, PBS $50 \mathrm{mM}(50 \mathrm{mM}$ phosphate buffer, $0.75 \mathrm{M} \mathrm{NaCl}, \mathrm{pH}$ 7), MES $0.1 \mathrm{M}$ (2-(N-morpholino) ethanesulfonic acid), HEPES (10 mM 4-(2-hydroxyethyl)piperazine1-ethanesulfonic acid, $300 \mathrm{mM} \mathrm{NaCl}, \mathrm{pH} 8$ ), Tween 20, dextran sulfate sodium salt (DS) (MW 40000 g mol-1), 16-mercaptohexadecanoic acid (MHDA), (1-ethyl-4(3dimethylaminopropyl)carbodiimide hydrochloride (EDC), ethanolamine and Nhydroxysulfosuccinimide (s-NHS)) were purchased from Sigma-Aldrich (Steinhem, Germany). Poly-L-lysine-graft-PEG (PLL-g-PEG) was purchased to SuSoS (Dübendorf, Switzerland). Commercial serum was obtained from Sigma-Aldrich (Steinhem, Germany). Recombinant RBD protein and its respective polyclonal IgG antibody (pAb-RBD) were obtained from 
SinoBiological (Eschborn, Germany). A polyclonal IgG antibody specific for N protein (pAbN) was purchased from GeneTex (Irvine, CA, US). WHO International Standard Anti-SARSCoV-2 immunoglobulin (code: 20/136) obtained from pooled plasma from individuals recovered from SARS-CoV-2 infection, was purchased from National Institute for Biological Standards and Control (NIBSC, Hertfordshire, United Kingdom). Monoclonal IgG antibody against CRP C7 (anti-CRP) was acquired from HyTest (Turku, Finland). The coding sequences of the $\mathrm{N}$ and C-terminal domains (positions 1-180 aa and 247-418 aa, respectively) of the SARS-CoV-2 N protein (Accession No. MT777677) were cloned in a pET 28a plasmid in fusion with a hexahistdine tag coding sequence at the 3 'end. Recombinant proteins were produced in E. coli T7 Express (DE3) cells (New England Biolabs) and purified under nondenaturing conditions by Immobilised Metal Affinity Chromatography followed by Size Exclusion Chromatography. Proteins were stored in $10 \mathrm{mM}$ HEPES, $150 \mathrm{mM} \mathrm{NaCl}, \mathrm{pH} 7.5$ buffer. $\mathrm{N}$ and $\mathrm{C}$-terminal domains were mixed in mass ratio $1 / 1$.

\subsection{SPR biosensor device}

The biosensor device employed is a homemade designed and assembled SPR that incorporates all the optical and microfluidic components in a compact and user-friendly platform $(20 \times 20$ $\mathrm{cm}^{2}$ ). The SPR sensor monitors the binding events in real-time by tracking the SPR-wavelength displacements $(\Delta \lambda, \mathrm{nm})$. It is based on the Kretschmann configuration and works at a fixed angle of incidence $\left(\theta=70^{\circ}\right)$. A description of the device is provided in the Supplementary Information (SI) and in the Figure S1. The device incorporates all fluidic components that allow the continuous flowing of solutions and the injection of samples $(150 \mu \mathrm{L})$. All the experiments were carried out in appropriate safety facilities, with the SPR biosensor located in a laboratory of Biosafety Level 2 (BSL-2).

\subsection{Plasmonic sensor chip preparation}

Gold plasmonic chips in-house fabricated ( $1 \mathrm{~nm} \mathrm{Ti} / 49 \mathrm{~nm} \mathrm{Au}$ ) were exposed to a surface cleaning procedure and chemically modified with alkanethiols (see Section 2 SI). Once properly cleaned, the sensor chips were placed on the SPR instrument for in-situ covalent immobilization of the viral proteins (N protein, RBD peptide or multianalyte (N+RBD. 1:1)) to carboxyl groups on the gold sensor chip through EDC/s-NHS chemistry ${ }^{33-35}$. For all the immobilized surfaces, a final blocking step was included to avoid non-specific adsorptions employing PLL-g-PEG ${ }^{36,37}$. Finally, the sensor chips were kept under a continuous flow of PBST+DS (PBS $10 \mathrm{mM}+0.5 \%$ Tween $20+2 \mathrm{mg} \mathrm{mL}^{-1} \mathrm{DS}$ ) at $15 \mu \mathrm{L} \mathrm{min}^{-1}$. Figure S1B shows a representative antigen immobilization of the three-step reaction involved in the covalent coupling to the gold sensor chip. 


\subsection{Antibody detection assays}

The experiments were performed with two different polyclonal antibodies, $\mathrm{pAb}-\mathrm{N}$ and $\mathrm{pAb}-$ $\mathrm{RBD}$, specific for $\mathrm{N}$ protein and the RBD domain, respectively, and with the first WHO International Standard Anti-SARS-CoV-2 human immunoglobulin. Real-time sensorgrams generated during the injection of the antibodies $(100 \mu \mathrm{L})$ were obtained in all the cases, monitoring the specific binding in each case (i.e. shift in the position of the resonance peak ( $\Delta \lambda$, $\mathrm{nm})$ to higher wavelengths). For single-antigen gold sensor chips, calibration curves were generated by analyzing different concentrations of the corresponding specific antibody (ranging from $100 \mathrm{ng} \mathrm{mL}^{-1}$ to $10 \mu \mathrm{g} \mathrm{mL}^{-1}$ ) in standard buffer (PBST+DS) or in commercial serum diluted to $10 \%$. For the RBD/N co-immobilized sensor chips, several mixtures of $\mathrm{pAb}-\mathrm{RBD}$ and $\mathrm{pAb}-$ $\mathrm{N}$ antibodies 1:1 at equal concentrations (from $100 \mathrm{ng} \mathrm{mL}^{-1}$ to $10 \mu \mathrm{g} \mathrm{mL}^{-1}$ ) were prepared in serum and analyzed after diluting at 10\%. Calibration curves were also generated employing the first WHO approved standard for serology assays, consisting of freeze-dried pooled plasma from eleven patients recovered from COVID-19 disease, whose stock solution has an assigned arbitrary unitage of $1000 \mathrm{BAU} \mathrm{mL}^{-1}$ (BAU, binding antibody units). Several concentrations were analyzed (ranging from 1.25 to $500 \mathrm{BAU} \mathrm{mL} \mathrm{m}^{-1}$ ) in standard buffer (PBST+DS) or in commercial serum diluted to $10 \%$ on the RBD/ $\mathrm{N}$ co-immobilized sensor chip. All the antibody solutions were injected over the sensor chip at a constant flow of $15 \mu \mathrm{L} \mathrm{min}{ }^{-1}$. In all the cases, antigen-antibody interaction was disrupted by injecting a $20 \mathrm{mM} \mathrm{NaOH}$ regeneration solution during $1 \mathrm{~min}$ at constant flow rate. Antigen-biofunctionalized plasmonic sensor chips could be reused between 15 and 20 times without altering or modifying the immobilized proteins and the assay performance.

\subsection{Data analysis}

The real-time sensorgrams were processed extracting the final response $(\Delta \lambda)$ after signal stabilization once the whole sample volume has passed through the flow cell. For the flow rate employed and the sample volume, this corresponds to approximately $1000 \mathrm{~s}$ after injection. The biosensor data were analyzed and processed using Origin 8.0 software (OriginLab Northampton, MA). Data and statistical analysis (one way ANOVA test) was performed using Graphpad Prism (Graphpad Software, Inc., California, US). Calibration curves were obtained by evaluating different concentrations of the polyclonal antibodies in triplicate. The mean sensor signal $(\Delta \lambda)$ and its standard deviation (SD) were plotted versus polyclonal antibody concentration. The data was fitted to a lineal regression equation $(\mathrm{y}=\mathrm{mX}+\mathrm{b})$ where $y$ is the sensor response, $X$ is the concentration of polyclonal antibody, $m$ is the slope of the linear regression curve and $b$ is the intercept. The limit of detection (LOD) for each antibody was calculated as the concentration corresponding to a blank signal plus three times its standard 
deviation. The coefficients of variation were obtained as the ratio of the standard deviation of the mean, expressed in percentages $(\% \mathrm{CV})$.

The differences between groups were analyzed with Kruskal-Wallis test, considering a $p$ value $p$ $<0.05$ to be statistically significant. Correlation between immune response and clinical severity was analyzed by Spearman test considering $\mathrm{p}$ value $<0.05$. Threshold values (cut-off values) to determine positive samples were calculated from the mean $+2 \mathrm{SD}$ of control negative samples. A value $<0.9 \mathrm{xMean}+2 \mathrm{SD}$ was considered negative; a value $>1.1 \mathrm{xMean}+2 \mathrm{SD}$ as positive; and between $0.9-1.1 x$ Mean $+2 \mathrm{SD}$ as indeterminate. The diagnostic sensitivity (SE), diagnostic specificity (SP), positive predictive value (PPV) and negative predictive value (NPV) were determined as described in the SI.

\subsection{Clinical samples collection}

A total of 125 clinical samples were collected from two hospitals in Barcelona (Spain) in three different batches. Two batches were provided by Vall d'Hebron University Hospital (VH.1 $\mathrm{n}=15$, and VH.2 $\mathrm{n}=70$ ), and a third batch was provided by the Clinic Hospital of Barcelona (CH.1 n=40). VH.1 and VH.2 serum samples and data from the patients used in this study were provided by the Clinical Microbiology Department and by the Sepsis Bank of Vall d'Hebron University Hospital Biobank (PT17/0015/0047), integrated in the Spanish National Biobanks Network. Samples were processed following standard operating procedures with the appropriate approval of the Clinical Research Ethics Committee (approval reference numbers for Vall d'Hebron University Hospital PR(AG)11/2016 and PR(AG297/2020)). VH.1 samples consisted of 15 serum samples from 15 different patients (5 negative pre-pandemic samples collected in 2016, and 10 COVID-19 positive samples, among which 5 were from patients with mild symptoms (i.e paucisymptomatic) and 5 from patients with severe symptoms (i.e. patients admitted to the ICU). VH.2 samples included 20 pre-pandemic negative samples and 50 COVID-19 positive samples, confirmed by PCR, ELISA and CLIA.

CH.1 serum samples included 40 COVID-19 positive samples that were collected by the Clinic Hospital of Barcelona following a symptomatology study. Samples included adult patients ( $>18$ years old) with SARS-CoV-2 symptomatology and with confirmed positive diagnosis during symptoms and confirmed negative diagnosis at infection resolution by PCR of nasopharyngeal swab, tracheal aspirate, or bronchoalveolar aspirate. Patients with HIV, hepatitis and immunosuppressed patients were excluded. Convalescent COVID-19 patients were cited to perform a rapid questionnaire of symptoms and confirm by LFA the presence of anti-SARSCoV-2 immunoglobulins. After this confirmation, a blood sample was obtained, and serum was processed and stored at a local biobank at $-80^{\circ} \mathrm{C}$ until analysis. Date of symptoms onset, symptoms description, Hospital or ICU admission and length of stay was analyzed in order to 
stratify patients according to severity: mild (symptomatic with no hospitalization), moderate (required hospital admission), and severe (required ICU admission) and symptomatology. The study was carried out in compliance with the Declaration of Helsinki (current version, Fortaleza, Brazil, October 2013) and was conducted in accordance with the requirements of the 2007 Spanish Biomedical Research Act. The study was approved by the institution's Internal Review Board (registry number $\mathrm{HCB} / 2020 / 0332$ ). Written informed consent was obtained from all patients.

\subsection{Standard analytical techniques (ELISA, CLIA and LFA)}

Serological response from the samples provided by Vall d'Hebron University Hospital (VH.1 and VH.2) was determined by ELISA and CLIA commercial kits. ELISA-IgG-S and ELISAIgA-S kits, which detect IgG and IgA antibodies against spike SARS-CoV-2 glycoprotein (AntiSARS-CoV-2 ELISA (IgG) (EUROIMMUN, Lübeck, Germany) were performed on the EUROIMMUN Analyzer I-2P (EUROIMMUN, Germany). ELISA tests have proven $96.9 \%$ and $94.4 \%$ sensitivity (SE) and $98.3 \%$ and $99.6 \%$ specificity (SP) for IgA and IgG, respectively. Samples resulting in cut-off index $(\mathrm{COI}) \geq 1.1$ were considered interpreted as positive. Besides, two different CLIA commercial kits were employed: (CLIA-1) CLIA-IgG-S kit: Liaison SARSCoV-2 S1/S2 IgG (DiaSorin, Italy), performed on the LIAISON® XL Analyzer (DiaSorin, Italy) for quantitative determination of the spike (S) glycoprotein subunits 1 and 2 (S1/S2) with $97.4 \%$ (SE) and $98.5 \%$ (SP). The result was considered positive when the COI $\geq 15 \mathrm{AU} / \mathrm{mL}$; (CLIA-2) ECLIA-Igs-N kit: Elecsys ${ }^{\circledR}$ Anti-SARS-CoV-2 (Roche Diagnostics, USA), performed on the Cobas 8800 system (Roche Diagnostics, USA) for qualitative determination of total antibodies (including IgG, IgM and IgA) against N SARS-CoV-2 protein with $100 \%$ (SE) and $99.80 \%$ (SP). The result was considered positive when COI $\geq 1.0$.

Lateral flow assays (LFA) were acquired from different suppliers: (LFA-1) 2019-nCoV IgG/IgM Detection Kit (Colloidal Gold-Based) Vazyme (Vazymebiotech Co, Nanjing, China) with 91.54\% (SE) and 97.02\% (SP); (LFA-2) Quick Profile 2019-nCoV IgG/IgM Test Card (Quick Profile, USA) with 87,8\% (SE) and 99\% (SP); (LFA-3) Wondfo Biotech (Guangzhou, Luogang, China) with $86.43 \%$ (SE) and $99.57 \%$ (SP); and (LFA-4) FaStep (Hangzhou, China) that identify $\operatorname{IgM}$ and $\operatorname{IgG}$ with $93.7 \%$ (SE) and $99.1 \%$ (SP). LFA tests were performed following the manufacturer's instructions. Briefly, a drop of blood or $10 \mu \mathrm{L}$ of serum and 2 drops of buffer solution were added to the corresponding loading area in the cassettes. After 15 min, the result was reflected by the appearance of colored bands. The LFA result was qualified based on the intensity of the Ig bands as follows: strong (3), regular (2) weak (1), and (0) no color change observed. 


\section{Results and discussion}

\subsection{Biosensor assay development and analytical characterization}

The in-house developed SPR biosensor platform employed monitors the shift in the position of the resonance peak of the plasmonic sensor chips, which reflects binding $(\Delta \lambda>0)$ or desorption events $(\Delta \lambda<0)$, being the signal proportional to the number of events (i.e. concentration). Our biosensor device has previously demonstrated its potential for clinical diagnostics in several areas, ${ }^{35,38}$ including infectious diseases ${ }^{34}$ and also for the direct detection of antibodies in human serum, ${ }^{33,36}$ enabling a one-step, label-free, and sensitive and reliable detection. These features are crucial to develop a fast test with response times below 15-20 minutes (thanks to the noneed of secondary reagents or further signal amplification steps), and with the potential of providing quantitative information (i.e., the concentration range of antibodies in serum).

In the case of COVID-19 serological assays, a key aspect to maximize both specificity and sensitivity is the viral antigen used for the detection of the antibodies. The $\mathrm{N}$ protein and the receptor binding domain (RBD) peptide contained in the spike protein appear to be both specially highly specific targets. ${ }^{11,15,39,40}$ Thus, we developed two different biofunctionalized sensor chips employing the $\mathrm{N}$ and RBD antigens, respectively, in order to capture the antibodies generated by the host.

To evaluate the performance of the biosensor-based assay, we employed commercial polyclonal antibodies for both $\mathrm{N}$ and RBD antigens, which can mimic the pool of antibodies with different antigen affinities produced by a host individual after infection. Figures $2 \mathbf{A}$ and $2 \mathbf{B}$ show representative detection signals (i.e., real-time sensorgrams) obtained for the two different biofunctionalized surfaces (i.e., $\mathrm{N}$ and RBD) with different pAb concentrations in buffer, gradually increasing as the concentrations were higher (i.e. $\Delta \lambda$ obtained after signal stabilization). A direct and linear relationship between the antibody concentration and the signal was observed (see Figures 2C and 2D) for the range of antibodies analyzed (i.e. from $100 \mathrm{ng}$ $\mathrm{mL}^{-1}$ to $10 \mu \mathrm{g} \mathrm{mL}^{-1}$ ), being possible to determine the limit of detection in both cases: $19.9 \mathrm{ng}$ $\mathrm{mL}^{-1}$ for anti-RBD immunoassay (slope $=0.2511 \mathrm{~nm} \mathrm{~mL} \mu \mathrm{g}^{-1}, \mathrm{R}^{2}=0.992$ ) and $45.6 \mathrm{ng} \mathrm{mL}^{-1}$ for anti-N (slope $=0.1536 \mathrm{~nm} \mathrm{~mL} \mu \mathrm{g}^{-1}, \mathrm{R}^{2}=0.994$ ). Some studies suggest that the antibody concentrations in COVID-19 patients' serum might lie in the range of $\mu \mathrm{g} \mathrm{mL} \mathrm{L}^{-1} 32$. According to these LODs, the performance of our biosensor provides enough analytical sensitivity for COVID-19 serological testing with both RBD and N-coated sensor chips.

The specificity was also evaluated in order to assure the absence of non-specific interactions of antibodies with the sensor chip surface. As Figures 2A and 2B show, neither pAb-N nor pAbRBD interacted with the opposite antigen surface (i.e., net sensor response after signal stabilization $\Delta \lambda=0 \mathrm{~nm}$ ), proving that no cross-reactivity between the antigen-antibody pairs was 
taking place. Similarly, a SARS-CoV-2 non-related antibody (i.e., anti-CRP) did not result in any signal, overall, confirming that the signals come exclusively from specific antigen-antibody interactions.

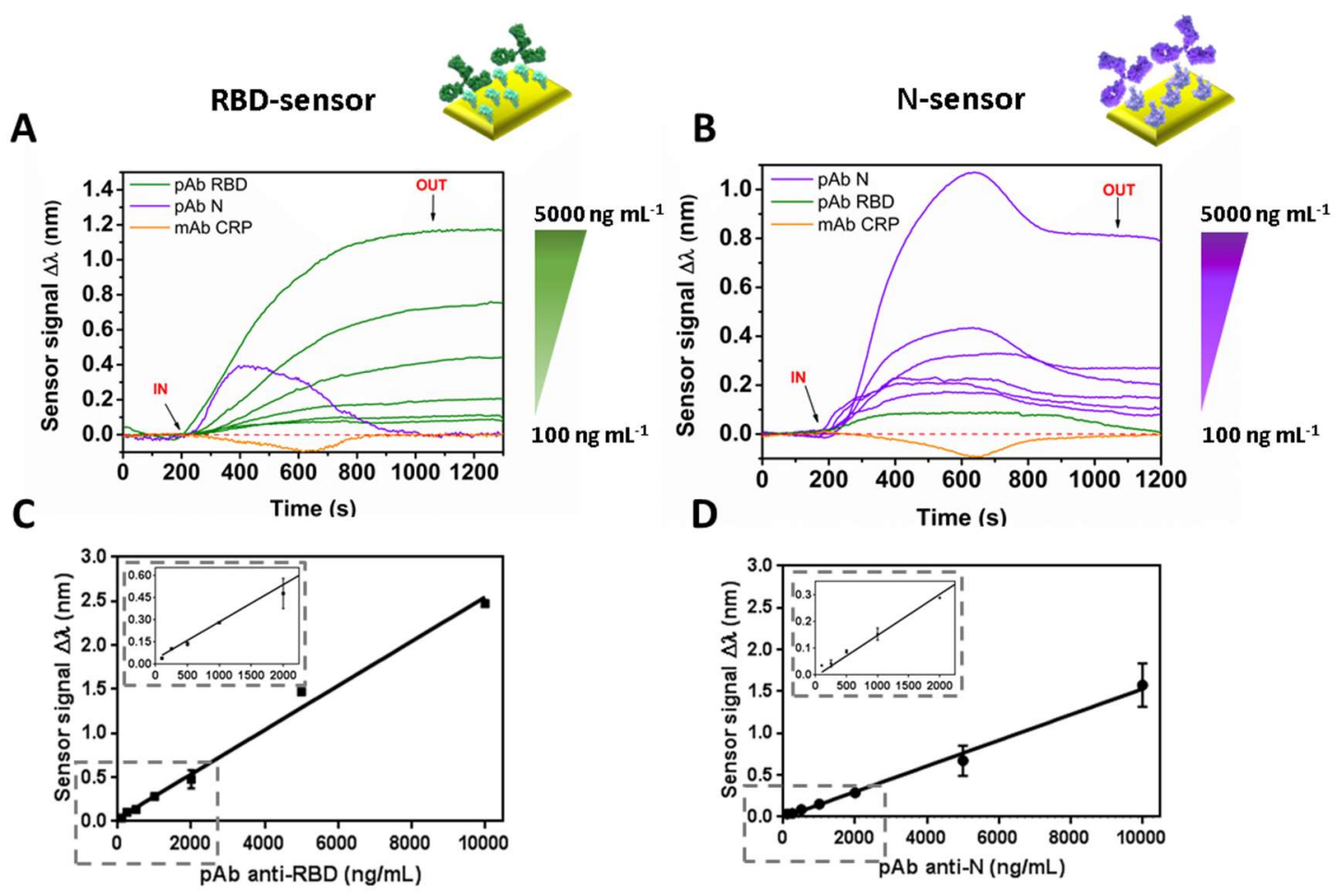

Figure 2. Real-time sensorgrams for different antibody concentrations over a (A) RBD-coated sensor chip and (B) N-coated sensor chip, in standard buffer conditions. Calibration curves in standard buffer for (C) RBD-coated sensors chips and (D) N-coated sensor chips, using the corresponding $\mathrm{pAb}$. Each signal corresponds to the mean $\pm \mathrm{SD}$ of triplicate measurements. Nonspecific antibodies were measured at a concentration of $2 \mu \mathrm{g} \mathrm{mL}^{-1}$.

In order to apply the described methodology in serological assays and therefore in patient's sera samples, we had to take into account the influence of the serum matrix on the sensor surface and the recognition event, as undiluted serum contains high amounts of proteins and other compounds that could generate non-specific interactions or hinder the protein-antibody interaction. For this reason, we decided to employ a combination of blocking agents including poly-L-Lysine grafted Poly(ethylene glycol) (PLL-g-PEG), detergent Tween 20 and sodium sulfate dextran salt, all of which have successfully reduced non-specific interactions in previous works. ${ }^{36,37}$

The performance of the assays in serum was directly evaluated with serum diluted at $10 \%$, as the detectability range of the assay might certainly tolerate this dilution (i.e., LOD in the $\mathrm{ng}^{-} \mathrm{L}^{-}$ ${ }^{1}$ range and presumably expected Ig concentrations in the $\mu \mathrm{g} \mathrm{mL^{-1 }}$ level) and still ensure a reliable semi- and quantitative detection. This dilution factor is considerably lower than the one commonly employed in ELISA or CLIA tests, which is around 40-200 times. ${ }^{32,41}$ In fact, under these conditions no undesired effects were observed for commercial serum, with negligible non- 
specific adsorptions and with a similarly wide dynamic range (see Figure 3A). The limit of detection achieved for the $\mathrm{N}$ biofunctionalized surface was twice higher than in standard buffer conditions (from $45.6 \mathrm{ng} \mathrm{mL}^{-1}$ to $86 \mathrm{ng} \mathrm{mL}^{-1}$ ), which might be related to a possible hindrance of the antibody-antigen interaction due to the serum matrix. However, RBD biofunctionalized surface exhibited a LOD of $21.1 \mathrm{ng} \mathrm{mL}^{-1}$, very similar to the obtained in standard buffer conditions. Under these conditions, both assays were further evaluated with real clinical samples.

In order to study the reproducibility of the assays in serum dilution, the inter-assay variability (replicates within different sensor chips) expressed as CV \% was studied. The values obtained for N-protein and RBD-domain were below to the maximum variability recommended for clinical analysis $(15 \%){ }^{42}$ (Table S1), overall confirming a good reproducibility and suitability of these viral antigens for polyclonal antibodies detection.

\subsection{Preliminary assessment of clinical samples}

We first evaluated a set of 15 clinical serum samples from 15 different patients (VH.1 collection consisting of 10 COVID-19 positive samples and 5 negative samples, collected in 2016 and stored in the Sepsis Bank of the Vall d'Hebron University Hospital Biobank). Positive serum samples were collected from patients previously diagnosed with COVID-19 by PCR, and who had a positive result of specific IgG and IgM class antibodies against the S1 subunit of SARSCoV-2, as described for the ELISA-IgG-S and ELISA-IgA-S. All the samples were analyzed with N-coated and RBD-coated sensor chips and a statistical comparison of both N-based and RBD-based serological assays was carried out. As can be seen in Figure 3B, the N-based assays showed poor differentiation between both sample groups, not being statistically significant $(p>$ 0.9999). RBD-based assay performed better as the $p$-value ( $p=0.0208)$, below 0.05 , indicates the discrimination between positive and negative samples does reach statistical significance. This result is in concordance with the respective calibration curves (Figure 3A) and the better sensitivity and detectability levels reached with the RBD-based assays. In addition, RBD-based assay shows less dispersion of the negative samples values compared to N-based assay, resulting in the absence of false negative (or indeterminate values). Table S1 compares RBDand N-based assays analytical parameters, where RBD-sensor shows a better sensitivity (slope $=0.261 \mathrm{~nm} \mathrm{~mL} \mu \mathrm{g}^{-1}, \mathrm{R}^{2}=0.999, \mathrm{LOD}=23.9 \mathrm{ng} \mathrm{mL}^{-1}$ ) than $\mathrm{N}$-sensor (slope $=0.0502 \mathrm{~nm} \mathrm{~mL}$ $\mu \mathrm{g}^{-1}, \mathrm{R}^{2}=0.9016, \mathrm{LOD}=80.3 \mathrm{ng} \mathrm{mL}^{-1}$ ). 
A

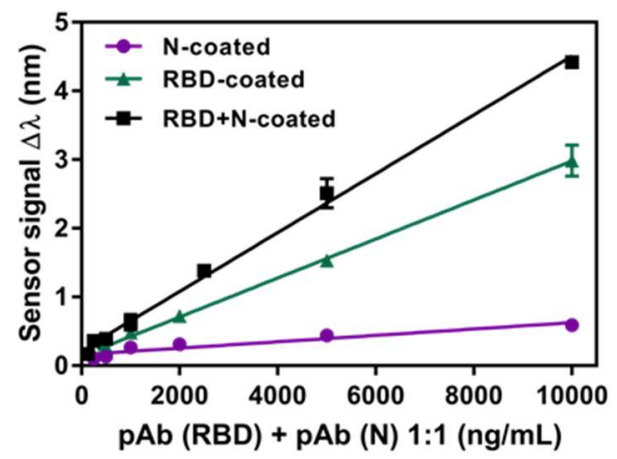

B

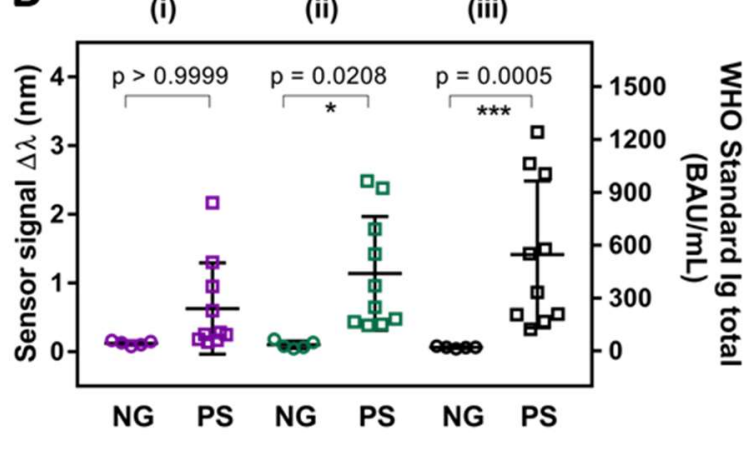

Figure 3. A) Calibration curves with $\mathrm{pAb}-\mathrm{N}$ and $\mathrm{pAb}-\mathrm{RBD}$ in $10 \%$ diluted commercial serum using three different biofunctionalized surfaces $(\mathrm{N}, \mathrm{RBD}$ and $\mathrm{RBD}+\mathrm{N})$. Sensor response represents the mean \pm SD of three measurements. B) Statistical comparison between the positive (PS) and negative (NG) clinical samples: (i) N-coated sensor chips; (ii) RBD-coated sensor chips; (iii) $\mathrm{RBD}+\mathrm{N}$-coated sensor chip. Kurskal-Wallis test $(\mathrm{p}=0.05)$. Total Ig concentration calculated from the WHO Standard Anti-SARS-CoV-2 immunoglobulin calibration curve is shown in the right axis.

In order to improve the discrimination between negative and positive samples, we assessed the performance of a serological assay employing a mixed sensor chip combining both the RBD and $\mathrm{N}$ antigens to capture antibodies targeting both proteins. Figure 3A shows that the multiantigen sensor surface significantly increased the detection signals of a mixture of both pAbs, reaching a better LOD than when using the antigens individually. The limit of detection was of $12.75 \mathrm{ng} \mathrm{mL}^{-1}$ with a slope of $0.475 \mathrm{~nm} \mathrm{~mL} \mu \mathrm{g}^{-1}\left(\mathrm{R}^{2}=0.997\right)$ and the calibration curve still shows a broad dynamic range (i.e., at high concentrations such as $10 \mu \mathrm{gL}^{-1}$ ) enabling the detection and quantification of antibodies even at high concentrations. Interestingly, as we can observe in Figure 3B, the analysis of real samples with the combined serological assay reveals higher responses, derived from the capture of both $\mathrm{N}$ and RBD antibodies. Moreover, the negative samples exhibit also lower signals than the single $\mathrm{N}$ - and RBD-based assays, reflecting more specificity, which reduces significantly the threshold and its standard deviation (see Figure 3B and Figure 4). From these factors, multianalyte surface is able to discriminate antiSARS-CoV-2 positive samples from negative samples with the most relevant statistical significance $(\mathrm{p}=0.0005)$, notably improving the performance of the individual antigen assays. The reproducibility study, which was done for viral antigens individually, was also performed for the multianalyte combination of RBD and $\mathrm{N}$ antigens (Table S1). As isolated antigens conditions, CV values related to multianalyte-assay were also below $15 \%$. This data supports the good reproducibility of the assay and the aptness of multianalyte condition for SARS-CoV-2 serological test. 


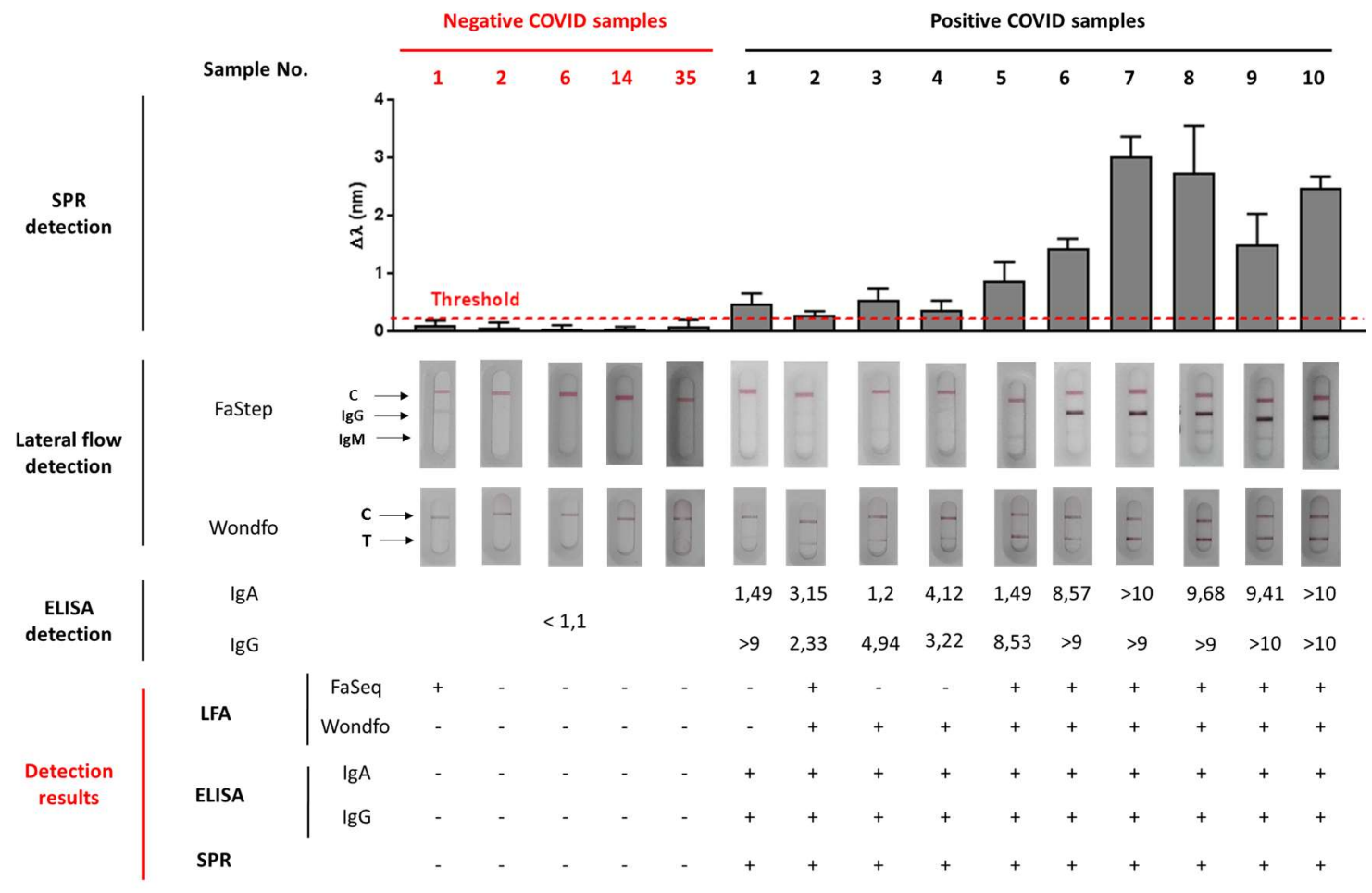

Figure 4. Performance comparison of different COVID-19 serological assays. SPR-biosensor assay, LFA tests and ELISA tests are shown for positives and negative serum samples. LFA tests were considered as positive after the appearance of a coloured band with regular (2) or strong (3) intensity in the IgG and/or IgM line, and negative for very weak (1) or not coloured bands (0). ELISA tests were considered positive for numeric values of IgG and/or IgA cut-off index $(\mathrm{COI})>1.1$. SPR biosensor assays were considered positive for samples above the set threshold (red dotted line) calculated as described in the experimental section. Detection results rows show the numbers of positive $(+)$ and negative $(-)$ samples for each serological methodology.

The RBD/N-based serological biosensor assay was qualitatively compared to standard ELISA done in clinics as well as to two different commercial lateral flow serological tests: Wondfo, which detect total Igs (against $\mathrm{S}$ protein) and FaStep, which detects both IgG and IgM against $\mathrm{N}$ and S1 proteins. We employed both lateral flow assays to analyze the 15 clinical samples. Results are summarized in Figure 4. The results obtained with an ELISA, which detects IgG and IgA antibodies against S1 protein, are also included. As can be observed, the SPR serological assay result precisely concurs to the commercial microplate-based assay, achieving promising sensitivity and specificity. Despite the ELISA does not provide quantitative information, a significant correlation between the relative numeric index obtained with this method (COI, cut-off index, extracted from the relative signal of the sample and a control calibrator) and the signal obtained with the SPR assay was observed for most of the samples, which might reflect the good accuracy of the biosensor assay. Interestingly, when analyzing the SPR quantitative detection results for COVID-19 positive samples, the values reveal a clear difference between two groups of samples, 1-5 and 6-10. The first set (1-5) corresponded to 
patients with mild symptomatology while samples 6-10 were obtained from ICU admitted patients. The SPR signals evidence higher levels of Ig for those patients with severe symptomatology compared with the ones with mild conditions. Moreover, LFA tests failed to identify some of those positive samples (i.e., FaStep 1, 3 and 4 and Wondfo 1) and wrongly identified as positive one negative sample (FaStep 1). These LFA results are in concordance with several systematic analysis which evidence deficient sensitivity and specificity of most LFA assays. ${ }^{16,17}$

Finally, in order to prove the quantification performance of the SPR biosensor and eventually facilitate its comparison with other serology assays detecting the same class of immunoglobulins, we carried out a calibration curve with the first WHO International Standard for Anti-SARS-CoV-2 immunoglobulin with the concentration expressed in arbitrary unitage of BAU $\mathrm{mL}^{-1}$. Calibration curves were generated in the same conditions as previously described for commercial pAb, in both standard buffer conditions (PBST $+\mathrm{DS}$ ) and $10 \%$ diluted serum. Figure 5 shows no differences between the calibration curves depending on conditions, achieving similar limits of detection, $0.098 \mathrm{BAU} \cdot \mathrm{mL}^{-1}$ for PBST+DS and $0.137 \mathrm{BAU} \cdot \mathrm{mL}^{-1}$ for diluted serum. According to this, patients' samples were analyzed and its immunoglobulin concentration was expressed in this standardized units.

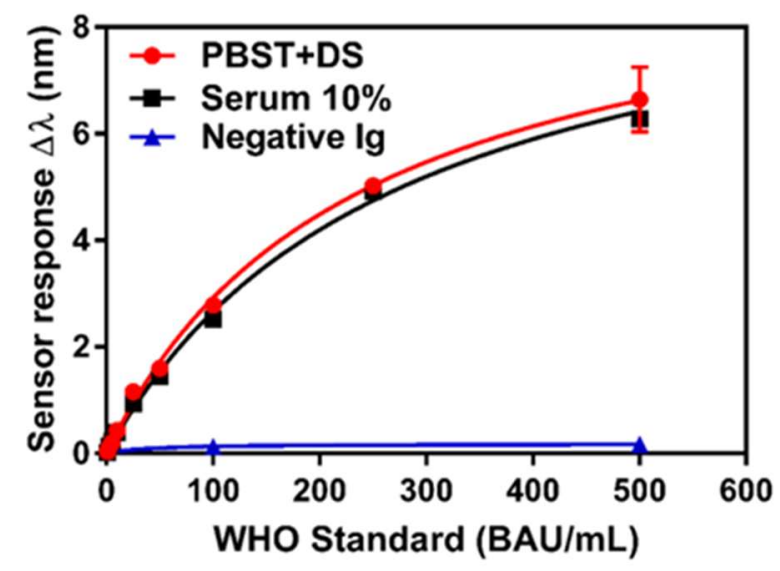

Figure 5. Calibration curves obtained using the WHO Standard anti-SARS-CoV-2 immunoglobulin in PBST + DS and $10 \%$ diluted commercial serum using $\mathrm{RBD}+\mathrm{N}$ biofunctionalized sensor surface. Biosensor response represents the mean $\pm \mathrm{SD}$ of three evaluations.

\subsection{Clinical validation of SPR-based COVID-19 serology}

Based on the results achieved with the preliminary clinical evaluation, a larger clinical validation study was initiated. A total of 120 clinical samples were analyzed, including 100 COVID-19 positive clinical samples collected during the pandemic with confirmed SARS-CoV2 PCR test and 20 negative samples collected prior the outbreak (Table 1 and Table S2). The serum samples collection was carried out between $>10$ days to months after the PCR results and 
they were assessed by the SPR biosensor as well as different commercial techniques such as ELISA, CLIA and LFA (details on the different tests employed in the experimental section and the SI).

Table 1. COVID-19 clinical samples classification/characterization

\begin{tabular}{|c|c|c|c|c|}
\hline & Total & Positive* & Negative & Characterization *" \\
\hline \multirow{2}{*}{$\begin{array}{l}\text { Vall d'Hebron } \\
\text { Hospital (VH) }\end{array}$} & \multirow{2}{*}{80} & \multirow{2}{*}{60} & \multirow{2}{*}{20} & $\begin{array}{l}\text { VH.1 }(n=10) \\
\text { ELISA }(10 / 10) \\
\text { LFA }(10 / 10)\end{array}$ \\
\hline & & & & $\begin{array}{l}\text { VH.2 }(n=50) \\
\text { CLIA }(50 / 50)\end{array}$ \\
\hline \multirow{2}{*}{$\begin{array}{l}\text { Clinic Hospital } \\
\text { (CH) }\end{array}$} & \multirow{2}{*}{40} & \multirow{2}{*}{40} & \multirow{2}{*}{0} & $\begin{array}{l}\text { CH.1 }(n=40) \\
\text { LFA }(40 / 40)\end{array}$ \\
\hline & & & & $\begin{array}{l}\text { mild }(n=14) \\
\text { moderate }(n=14) \\
\text { severe }(n=12)\end{array}$ \\
\hline
\end{tabular}

${ }^{*}$ Samples from patients with a positive PCR

${ }^{* *}$ Characterization of positive samples is summarized in the Table S2 (SI)

Figure 6 shows the distribution of the sensor signal obtained employing our biosensor assay for each of the samples. Positive samples showed a variable distribution of Ig levels which might go from few BAU $\cdot \mathrm{mL}^{-1}$ to thousands of $\mathrm{BAU} \cdot \mathrm{mL}^{-1}$. Threshold value was determined from previous assessment study employing confirmed negative serum samples obtained before the COVID-19 pandemic (Table S3). All the 20 negative samples studied gave signals below the threshold, while only one of the PCR positive samples was considered not positive (indeterminate). The SPR-based serological test shows a sensitivity and PPV of 99\% and 100\%, respectively. On the other hand, it was able to discriminate negative cases, with a specificity and NPV of $100 \%$ both.

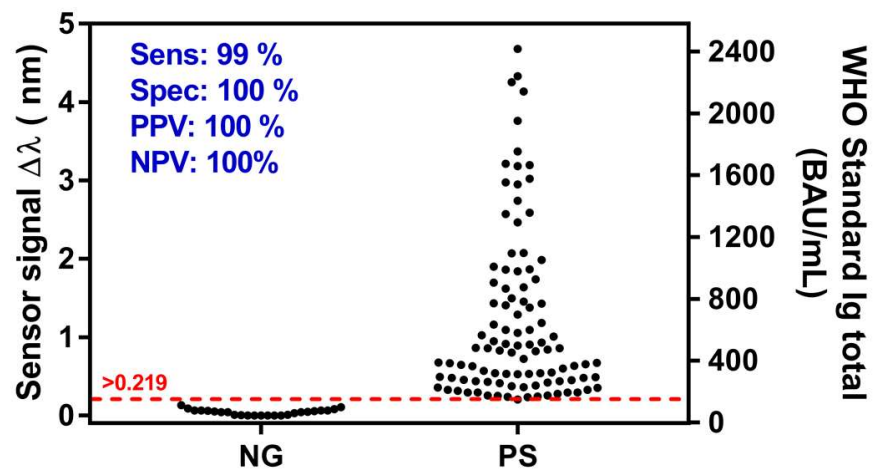

Figure 6. Sensor signal distribution of 100 COVID-19 positive (PS) and 20 negative (NG) clinical samples. Total Ig concentration calculated from the WHO Standard calibration curve is shown in the right axis. Sensitivity, specificity, PPV, NPV and threshold are also shown. 
To evaluate and validate the accuracy of our SPR serological biosensor, we compared our results with the methods and techniques employed in the two Hospitals. Commercial LFAs employed in this study report sensitivities between $90-95 \%$. On the other hand, for standard clinical techniques like CLIA and ELISA, sensitivities usually exceed 95\%, reaching 100\% in some cases. All cited methodologies have reported specificities between 97-99.8\%. Table 2 summarizes the diagnostic results obtained for the whole collection of COVID-19 positive samples (VH.1, VH.2, and CH.1) when analyzed by ELISA, CLIA, LFAs and our SPR biosensor. LFA results, classified according to the intensity scale described in the experimental section (i.e., 0-no visible color change, 1-weak, 2-regular, and 3-strong) were categorized as negative (intensity 0), indeterminate (intensity 1 ) and positive (intensity 2 and 3). ELISA, CLIA and SPR results where categorized depending on the determined threshold for each technique (Table S3 provides all data obtained with each technique).

Table 2. Summary of COVID-19 clinical samples validation

\begin{tabular}{|c|c|c|c|c|c|}
\hline$V H .1$ & $P C R$ & $S P R$ & $\begin{array}{c}\text { ELISA } \\
\text { Euroimmun }\end{array}$ & $\begin{array}{c}\text { LFA } \\
\text { Wondfo }\end{array}$ & $\begin{array}{c}\text { LFA } \\
\text { FaStep }\end{array}$ \\
\hline Positive & 10 & 10 & 10 & 9 & 7 \\
\hline Indeterminate & 0 & 0 & 0 & 0 & 2 \\
\hline Negative & 0 & 0 & - & 1 & 1 \\
\hline VH. 2 & $P C R$ & SPR & $\begin{array}{c}\text { CLIA } \\
\text { Liaison }\end{array}$ & $\begin{array}{c}\text { CLIA } \\
\text { Elecsys }\end{array}$ & \\
\hline Positive & 50 & 49 & 46 & 48 & \\
\hline Indeterminate & 0 & 1 & $4^{*}$ & 0 & \\
\hline Negative & 0 & 0 & 0 & 2 & \\
\hline CH.1 & PCR & $S P R$ & $\begin{array}{c}\text { LFA } \\
\text { Vazyme }\end{array}$ & $\begin{array}{c}\text { LFA } \\
\text { Quick Profile }\end{array}$ & \\
\hline Positive & 40 & 40 & 18 & 18 & \\
\hline Indeterminate & 0 & 0 & 4 & 4 & \\
\hline Negative & 0 & 0 & 18 & 18 & \\
\hline
\end{tabular}

*ELISA (Euroimmun) was performed to confirm indeterminate results

In views of the results of Table 2, we can affirm that our SPR biosensor outperforms the different approved diagnostic techniques employed in this study for the number of samples tested. The SPR biosensor can provide highly accurate detection of COVID-19 antibodies in a 15 min assay time (same as LFA), with a diagnostic sensitivity, specificity and reliability equivalent to ELISA and CLIA. Therefore, we herein have demonstrated the major benefit promised by label-free plasmonic biosensor technology: simple, rapid, and reliable diagnostics.

\subsection{Relationship between humoral immunity in SARS-CoV-2 infection and clinical severity}

To test the capabilities of the SPR biosensor for quantitative assessment of acquired immunity, a preliminary study was carried out to ascertain the existence of a possible correlation between the 
severity outcome and the levels of SARS-CoV-2 antibodies in sera (as seemingly observed in the preliminary assessment). The study was performed with a set of samples after a daily screening in the Clinic Hospital of Barcelona. Patients with SARS-CoV-2 antibodies were confirmed by LFA, identifying the presence of IgG and IgM anti-SARS-CoV-2. To stratify patients according to severity and symptomatology date of symptoms onset, symptoms description, Hospital or ICU admission and length of stay were analyzed. Finally, 40 serum samples from convalescent COVID patients with diverse severity (mild $(n=14)$, moderate $(n=14)$ and severe $(n=12))$ were included on the validation assay with the plasmonic biosensor. All included patients were symptomatic without statistical difference on symptoms between groups. In brief, the frequency of each symptom by mild, moderate or severe was respectively: fever $(71 \%, 79 \%$ and $100 \%, \mathrm{p}=1.43)$, myalgias $(57 \%, 14 \%$ and $33 \%, \mathrm{p}=0.059)$, cough $(57 \%$, $57 \%, 67 \%, \mathrm{p}=0.853)$, anosmia $(50 \%, 36 \%, 42 \%, \mathrm{p}=0.745)$, ageusia $(36 \%, 21 \%, 33 \%, \mathrm{p}=0.680)$, diarrhea $(21 \%, 36 \%, 50 \%, \mathrm{p}=0.313)$, headache $(21 \%, 39 \%, 33 \%, \mathrm{p}=0.790)$, dyspnea $(21 \%$, $36 \%, 58 \%, \mathrm{p}=0.151)$. The time since symptoms onset until samples collection differed between groups (in days) 52.00[44.75-63.25], 76.00 [67.00-88.00] and 118.50[73.50-123.75], $\mathrm{p}<0.0001$, respectively (Figure S2). This was attributed to a more deteriorated health status in severe patients who needed more recovery time from symptoms onset to the inclusion visit. ${ }^{43}$

Although LFA showed limited sensitivity for the detection of anti-SARS-CoV-2 antibodies in some cases, especially in mild severity patients, a correlation was detected between intensity of the LFA and COVID severity. Related to IgG, $85.7 \%$ of moderate and $83.3 \%$ of severe patients had positive LFA for IgG ( $\mathrm{p}=000.1$ ), whereas $100 \%$ of mild patients had negative LFA result for IgG. As regards IgM, $100 \%$ of mild and $93 \%$ of moderate patients had negative LFA results but $42 \%$ of severe patients had positive LFA for $\operatorname{IgM}(\mathrm{p}=0.007)$. Both, IgG and IgM were presented only in severe patients, showing in IgG case, higher intensity of the line LFA result than moderate cases (Table S2). Contrarily, no immunoglobulins were detected in mild patients. Thus, LFA assays showed a possible association between humoral immunology response and clinical severity due to SARS-CoV-2 infection.

In contrast to LFA, for all patients, regardless of the severity group, we detected immunoglobulins levels with the plasmonic biosensor. The antibody levels were more elevated in the moderate and severe groups versus the mild one. However, the levels of immunoglobulins did not differ statistically between groups (0.87[0.36-3.02], 1.44[0.50-1.83] and 1.07[0.921.80]]) $\mathrm{p}=0.548$ (Figure 7). In addition, we did not find correlation between levels of antibodies and COVID-19 symptomatology and severity $(\mathrm{r}=0.175$ and $\mathrm{p}=0.279)$. 


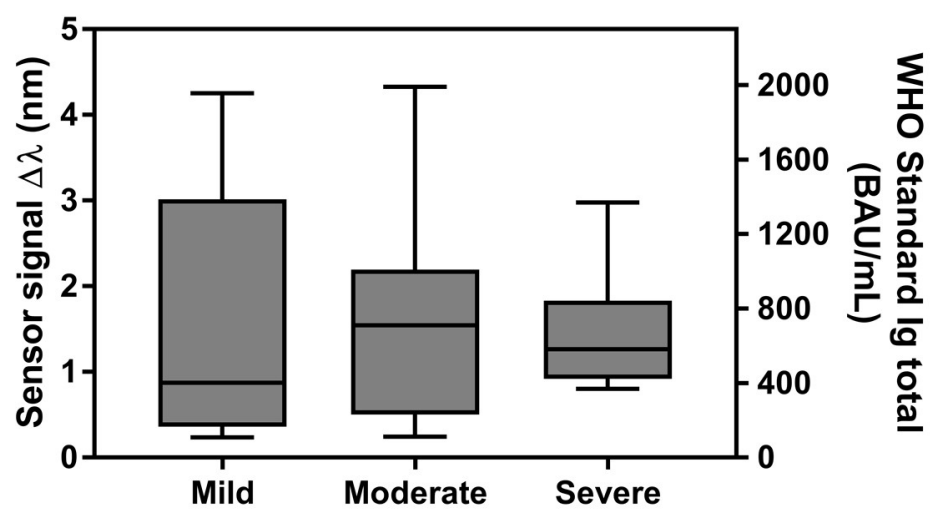

Figure 7. Correlation outcome severity $v s$ antibodies concentration. Sensor signal of 40 COVID-19 positive samples from individuals with different degrees of severity (mild, moderate and severe symptoms). Spearman test $(\mathrm{p}=0.05)$. Total $\mathrm{Ig}$ concentration calculated from the WHO Standard calibration curve is shown in the right axis

From this preliminary study, and with the limited pool of samples analyzed, we can assert that the SPR biosensor technology shows high sensitivity for identifying total SARS-CoV-2 immunoglobulins but so far it does not provide conclusive information regarding a possible correlation between the severity degree and immunity response reflected as SARS-CoV-2 antibodies concentration in serum. There is not still a consensus in bibliography about the relationship between humoral immunity response and severity outcome after SARS-CoV-2 infection. Some publications state that, as Middle East Respiratory Syndrome (MERS) virus infection, it exists a strong association. ${ }^{44-47}$ Nevertheless, others publications cannot confirm this relationship, showing similar neutralizing antibodies in both cohorts, critical and non-critical COVID-19 patients. ${ }^{48-50}$ The studies differences related to number of samples, the time since symptoms onset until samples collection, and others factor as the limitations of each study, could lead to the current controversies. Despite we acknowledge the necessity of completing the study with an extended number of samples and a longitudinal study, this pilot study exemplifies the convenience a serological quantitative assay may provide to monitor immune response evolution, even for early samples, with still IgM present in the serum.

\section{Conclusions}

We have demonstrated and fully validated a new biosensor technology, based on SPR, for rapid - less than $15 \mathrm{~min}$ - identification and quantification of total SARS-CoV-2 antibodies in blood serum. Different strategies were explored depending on the antigen selected to identify SARS$\mathrm{CoV}-2$ antibodies (N protein and RBD peptide) achieving the most sensitive and specific results when combining RBD and N antigens onto the SPR sensor chip surface. The multianalyte-based biosensor reached outstanding limits of detection in serum (low $\mathrm{ng} \mathrm{mL}^{-1}$ range) that enable direct one-step detection and quantification of SARS-CoV-2 antibodies in COVID-19 patients, and showing an excellent discrimination between positive and negative samples $(\mathrm{p}=0.0005)$. 
Moreover, we have implemented the biosensor assay with the first approved Anti-SARS-CoV-2 Immunoglobulin Standard that will allow further comparison with other serological assays.

We have completed an extended clinical validation with COVID-19 positive and negative samples $(n=120)$ that demonstrates a diagnostic sensitivity of $99 \%$ and diagnostic specificity of $100 \%$ of our biosensor, outperforming current available techniques like immunoassays and rapid tests. We have also done a preliminary study of correlation between the humoral immune response and the clinical severity outcome, although a higher cohort would be necessary to generate more conclusive information.

Overall, the results obtained position our biosensor device as an accurate, robust, and easy-touse tool for rapid and reliable COVID-19 serology to be employed both at laboratory and decentralized settings. In addition, the biosensor platform is user-friendly and miniaturized, paving the way to a smooth technological transfer. This work further illustrates the large versatility that SPR biosensors account to be readily adapted to the detection of different types of target biomarkers, thereby becoming a potential alternative tool for rapid diagnostics with great perspectives in clinical practice implementation.

\section{Acknowledgements}

ICN2 and UVE acknowledge financial support from H2020 Research and Innovation Programme of the European Commission (H202-SC1-PHE-CORONAVIRUS-2020, CONVAT Project, No. 101003544). The ICN2 is funded by the CERCA program/Generalitat de Catalunya and supported by the Severo Ochoa Centres of Excellence program funded by the Spanish Research Agency (AEI, grant no. SEV-2017-0706). ICN2 group is very grateful to EPI Industries (Barcelona, Spain) for its kind donation supporting our research in COVID-19. O.CL. acknowledges the economic support from the Spanish Ministry of Science and Innovation and the Spanish Research Agency and the European Social Fund (ESF) (ref. BES-2017-080527) linked to the TEC 2016-78515-R project PREDICT. A part of the work was supported by the European Virus Archive GLOBAL (EVA-GLOBAL) project that has received funding from the EU Horizon 2020 (grant agreement No. 871029). A.T. and L.F-B. acknowledge financial support from GENCAT-DGRIS COVID. We are indebted to all the patients who accepted to participate contributing to science advancement. We are indebted to the HCB-IDIBAPS Biobank for the human samples and data procurement and to the Fundació Glòria Soler for its support to the COVIDBANK collection. We thank the IDIBAPS Biobank for its valuable contribution to sample processing and storage. The authors acknowledge the EU Horizon 2020 Program under grant agreement no. 644956 (RAIS project) for funding the Hospital Vall d'Hebron Biobank. The VHIR-HUVH is supported by Plan Nacional de I+D+i 2013-2016 and ISCIII- Ministerio de Ciencia e Innovación, and Spanish Network for Research in Infectious 
Diseases (REIPI RD16/0016/0003) — cofinanced by European Development Regional Fund "A way to achieve Europe," Operative program Intelligent Growth 2014. Part of the samples and data from patients included in this study were provided by Vall d'Hebron University Hospital Biobank (PT17/0015/0047), integrated in the Spanish National Biobanks Network, and they were processed following standard operating procedures with the appropriate approval of the Ethical and Scientific Committee. The authors kindly appreciate the generous donation of samples and clinical data of the donors of the Sepsis Bank of HUVH Biobank and COVID-19 patients attended at HUVH.

\section{Author contribution}

O.C-L. and M.Si. carried out the optimization of the assay and the SPR measurements. S.A., R.C., and B.C. carried out the synthesis of the nucleocapsid viral antigen. L.C-C., A.R-S., J.C.R-R., R.F., J.J.G-L., J.E., and C. F-N. collected, treated, and validated the clinical samples from Vall D'Hebron Hospital and L.B., R.L-A, A.T., L.F-B. from Clinic Hospital. O.C-L., M.Si., M.S., and M-C.E. conceptualized the experiments and wrote the presented manuscript. L.F-B., J.J.G-L., J.E., J.C.R-R., B.C. and L.M.L. took a part in the discussion of the following manuscript and revised it critically for important intellectual content. All authors have read and agreed to the published version of the manuscript 


\section{References}

1. COVID-19 Map - Johns Hopkins Coronavirus Resource Center. https://coronavirus.jhu.edu/map.html.

2. Hu, B., Guo, H., Zhou, P. \& Shi, Z. L. Characteristics of SARS-CoV-2 and COVID-19. Nature Reviews Microbiology 1-14 (2020) doi:10.1038/s41579-020-00459-7.

3. Wu, D., Wu, T., Liu, Q. \& Yang, Z. The SARS-CoV-2 outbreak: What we know. International Journal of Infectious Diseases vol. 94 44-48 (2020).

4. Wiersinga, W. J., Rhodes, A., Cheng, A. C., Peacock, S. J. \& Prescott, H. C. Pathophysiology, Transmission, Diagnosis, and Treatment of Coronavirus Disease 2019 (COVID-19): A Review. JAMA - Journal of the American Medical Association vol. 324 782-793 (2020).

5. Zhang, N. et al. Recent advances in the detection of respiratory virus infection in humans. J. Med. Virol. 92, 408-417 (2020).

6. Hasell, J. et al. A cross-country database of COVID-19 testing. Sci. Data 7, 1-7 (2020).

7. Nagura-Ikeda, M. et al. Clinical evaluation of self-collected saliva by quantitative reverse transcription-PCR (RT-qPCR), Direct RT-qPCR, reverse transcription-loopmediated isothermal amplification, and a rapid antigen test to diagnose COVID-19. J. Clin. Microbiol. 58, (2020).

8. Hirotsu, Y. et al. Comparison of automated SARS-CoV-2 antigen test for COVID-19 infection with quantitative RT-PCR using 313 nasopharyngeal swabs, including from seven serially followed patients. Int. J. Infect. Dis. 99, 397-402 (2020).

9. Hsueh, P. R., Huang, L. M., Chen, P. J., Kao, C. L. \& Yang, P. C. Chronological evolution of IgM, IgA, IgG and neutralisation antibodies after infection with SARSassociated coronavirus. Clin. Microbiol. Infect. 10, 1062-1066 (2004).

10. Woo, P. C. Y. et al. Longitudinal profile of immunoglobulin G (IgG), IgM, and IgA antibodies against the severe acute respiratory syndrome (SARS) coronavirus nucleocapsid protein in patients with pneumonia due to the SARS coronavirus. Clin. Diagn. Lab. Immunol. 11, 665-668 (2004).

11. Long, Q. X. et al. Antibody responses to SARS-CoV-2 in patients with COVID-19. Nat. Med. 26, 845-848 (2020).

12. Qu, J. et al. Profile of Immunoglobulin G and IgM Antibodies Against Severe Acute Respiratory Syndrome Coronavirus 2 (SARS-CoV-2). Clin. Infect. Dis. 71, 2255-2258 
(2020).

13. Krammer, F. \& Simon, V. Serology assays to manage COVID-19. Science 368, 1060 $1061(2020)$.

14. Leung, G. M. et al. Seroprevalence of IgG antibodies to SARS-coronavirus in asymptomatic or subclinical population groups. Epidemiol. Infect. 134, 211-221 (2006).

15. Carter, L. J. et al. Assay Techniques and Test Development for COVID-19 Diagnosis. ACS Cent. Sci. 6, 591-605 (2020).

16. Whitman, J. D. et al. Evaluation of SARS-CoV-2 serology assays reveals a range of test performance. Nat. Biotechnol. 38, 1174-1183 (2020).

17. Mekonnen, D. et al. Diagnostic accuracy of serological tests and kinetics of severe acute respiratory syndrome coronavirus 2 antibody: A systematic review and meta-analysis. Rev. Med. Virol. e2181 (2020) doi:10.1002/rmv.2181.

18. Rashid, Z. Z., Othman, S. N., Samat, M. N. A., Ali, U. K. \& Wong K. K. Diagnostic performance of COVID-19 serology assays. Malays J Pathol. 42, 13-21 (2020).

19. Lisboa Bastos, M. et al. Diagnostic accuracy of serological tests for covid-19: Systematic review and meta-analysis. BMJ 370, 2516 (2020).

20. Grifoni, A. et al. Targets of T Cell Responses to SARS-CoV-2 Coronavirus in Humans with COVID-19 Disease and Unexposed Individuals. Cell 181, 1489-1501.e15 (2020).

21. Zhang, X. et al. Viral and host factors related to the clinical outcome of COVID-19. Nature 583, 437-440 (2020).

22. Masson, J. F. Surface Plasmon Resonance Clinical Biosensors for Medical Diagnostics. ACS Sensors vol. 2 16-30 (2017).

23. Soler, M., Huertas, C. S. \& Lechuga, L. M. Label-free plasmonic biosensors for pointof-care diagnostics: a review. Expert Review of Molecular Diagnostics vol. 19 71-81 (2019).

24. Kumbhat, S., Sharma, K., Gehlot, R., Solanki, A. \& Joshi, V. Surface plasmon resonance based immunosensor for serological diagnosis of dengue virus infection. J. Pharm. Biomed. Anal. 52, 255-259 (2010).

25. Jahanshahi, P., Zalnezhad, E., Sekaran, S. D. \& Adikan, F. R. M. Rapid immunoglobulin M-based dengue diagnostic test using surface plasmon resonance biosensor. Sci. Rep. 4, $1-7$ (2014). 
26. Jongerius-Gortemaker, B. G. M., Goverde, R. L. J., Van Knapen, F. \& Bergwerff, A. A. Surface plasmon resonance (BIACORE) detection of serum antibodies against Salmonella enteritidis and Salmonella typhimurium. J. Immunol. Methods 266, 33-44 (2002).

27. Riedel, T. et al. Diagnosis of Epstein-Barr virus infection in clinical serum samples by an SPR biosensor assay. Biosens. Bioelectron. 55, 278-284 (2014).

28. Park, T. J., Hyun, M. S., Lee, H. J., Lee, S. Y. \& Ko, S. A self-assembled fusion proteinbased surface plasmon resonance biosensor for rapid diagnosis of severe acute respiratory syndrome. Talanta 79, 295-301 (2009).

29. Soler, M., Estevez, M. C., Cardenosa-Rubio, M., Astua, A. \& Lechuga, L. M. How Nanophotonic Label-Free Biosensors Can Contribute to Rapid and Massive Diagnostics of Respiratory Virus Infections: COVID-19 Case. ACS Sensors 5, 2663-2678 (2020).

30. Ruiz-Vega, G., Soler, M. \& Lechuga, L. M. Nanophotonic biosensors for point-of-care COVID-19 diagnostics and coronavirus surveillance. JPhys Photonics vol. 311002 (2021).

31. Djaileb, A. et al. A rapid and quantitative serum test for SARS-CoV-2 antibodies with portable surface plasmon resonance sensing. ChemRxiv (2020) doi:10.26434/chemrxiv.12118914.v1.

32. Ma, H. et al. COVID-19 diagnosis and study of serum SARS-CoV-2 specific IgA, IgM and $\mathrm{IgG}$ by chemiluminescence immunoanalysis. medRxiv 2020.04.17.20064907 (2020) doi:10.1101/2020.04.17.20064907.

33. Soler, M. et al. Highly sensitive dendrimer-based nanoplasmonic biosensor for drug allergy diagnosis. Biosens. Bioelectron. 66, 115-123 (2015).

34. Peláez, E. C. et al. Detection and Quantification of HspX Antigen in Sputum Samples Using Plasmonic Biosensing: Toward a Real Point-of-Care (POC) for Tuberculosis Diagnosis. ACS Infect. Dis. 6, 1110-1120 (2020).

35. Peláez, E. C. et al. A compact SPR biosensor device for the rapid and efficient monitoring of gluten-free diet directly in human urine. Anal. Bioanal. Chem. 412, 64076417 (2020).

36. Soler, M., Estevez, M. C., Villar-Vazquez, R., Casal, J. I. \& Lechuga, L. M. Label-free nanoplasmonic sensing of tumor-associate autoantibodies for early diagnosis of colorectal cancer. Anal. Chim. Acta 930, 31-38 (2016). 
37. Peláez, E. C. et al. Nanoplasmonic biosensor device for the monitoring of acenocoumarol therapeutic drug in plasma. Biosens. Bioelectron. 119, 149-155 (2018).

38. Calvo-Lozano, O. et al. Fast and accurate pneumocystis pneumonia diagnosis in human samples using a label-free plasmonic biosensor. Nanomaterials 10, 1-18 (2020).

39. Premkumar, L. et al. The receptor-binding domain of the viral spike protein is an immunodominant and highly specific target of antibodies in SARS-CoV-2 patients. Sci. Immunol. 5, (2020).

40. Burbelo, P. D. et al. Sensitivity in Detection of Antibodies to Nucleocapsid and Spike Proteins of Severe Acute Respiratory Syndrome Coronavirus 2 in Patients With Coronavirus Disease 2019. J. Infect. Dis. 222, 206-213 (2020).

41. $\mathrm{Hu}, \mathrm{X}$. et al. Impact of heat-inactivation on the detection of SARS-CoV-2 IgM and IgG antibody by ELISA. Clin. Chim. Acta 509, 288-292 (2020).

42. FDA, U. D. of H. and H. S. F. and D. A. C. for D. E. and R. and C. for V. M. Bioanalytical method validation guidance for industry. https://ci.nii.ac.jp/naid/10025804295/ (2018).

43. Fernández-Barat, L., López-Aladid, R. \& Torres, A. The value of serology testing to manage SARS-CoV-2 infections. European Respiratory Journal vol. 56 (2020).

44. Zhao, J. et al. Antibody Responses to SARS-CoV-2 in Patients With Novel Coronavirus Disease 2019. Clin. Infect. Dis. 71, 2027-2034 (2020).

45. Okba, N. M. A. et al. Severe Acute Respiratory Syndrome Coronavirus 2-Specific Antibody Responses in Coronavirus Disease Patients. Emerg. Infect. Dis. 26, 1478-1488 (2020).

46. Edouard, S. et al. Evaluating the serological status of COVID-19 patients using an indirect immunofluorescent assay, France. Eur. J. Clin. Microbiol. Infect. Dis. 40, 361$371(2021)$.

47. Garcia-Beltran, W. F. et al. COVID-19-neutralizing antibodies predict disease severity and survival. Cell 184, 476-488.e11 (2021).

48. Yongchen, Z. et al. Different longitudinal patterns of nucleic acid and serology testing results based on disease severity of COVID-19 patients. Emerg. Microbes Infect. 9, 833$836(2020)$.

49. GeurtsvanKessel, C. H. et al. An evaluation of COVID-19 serological assays informs future diagnostics and exposure assessment. Nat. Commun. 11, 1-5 (2020). 
50. Phipps, W. S. et al. SARS-CoV-2 Antibody Responses Do Not Predict COVID-19 Disease Severity. Am. J. Clin. Pathol. 154, 459-465 (2020). 